EPiC Series in Education Science
Volume 3, 2020, Pages 214-223
Proceedings of the MIT LINC 2019 Conference

\title{
Can virtual reality act as an affective machine? The wild animal embodiment experience and the importance of appearance
}

\author{
Alexandra Sierra Rativa ${ }^{1}$ Marie Postma ${ }^{1}$ and Menno van Zaanen ${ }^{1}$ \\ ${ }^{1}$ Department of Cognitive Science and Artificial Intelligence, Tilburg University \\ Tilburg, The Netherlands \\ aasierrar@uvt.nl, bmarie.postma@uvt.nl, mvzaanen@uvt.nl
}

\begin{abstract}
In view of the growing urgency to protect wildlife, the general goal of our research is to develop an immersive virtual experience where users can step into the 'shoes' of wild animals. The specific objective of this research is to explore the possibility of creating a strong emotional connection experience with a virtual animal body. In a game setting, users explore a simulated natural habitat of the animal. At the end of the game, users experience a distress event during which they become the target of an illegal animal hunter. The users receive physical feedback through haptic virtual reality suits (vibrating motors) that mimic the sensation of feeling pain of a hunter's shot. We compare the perceived pain, empathy, immersion, and embodiment experience evoked through a game character with a natural body (beaver), with an artificial body (robot beaver), and an amorphous body. The results of this investigation show a significant effect of game character appearance and perceived pain during the distress event. Moreover, we find a significant effect of game character appearance on immersion. These results suggest that the design of the game character appearance can influence users' emotional connectedness to the character and the game experience.
\end{abstract}

Keywords: virtual reality, empathy, immersion, virtual animal, virtual robot animal, animal conservation, perceived-pain, natural body, artificial body, beaver, robot beaver, game character appearance, emotions.

\section{Introduction}

Recently, it has been reported that approximately between 1 to 5 species go extinct each year (Earthday.org., 2018). Ultimately, half of all wild animal species are expected to become extinct by the end of this century. Many environmental organizations significantly contribute to the protection of wild animals; however, the problem persists and has become increasingly dire each year. Large scale efforts to alleviate the plight of wild animals seem to be hampered by the limited emotional connection to 
nature (Wilson, 2008). Since most people live in large cities and have no direct contact with wildlife, they feel disconnected from the problems of biodiversity and conservation (Miller, 2005).

Educators around the world have used technological media such as videos, games, and other resources to discuss protection of endangered species in the classroom. Although large numbers of students are aware of the extinction of wild animals, this does not seem to have a direct impact on the empathy and emotional connection towards these animals. How can we increase the emotional connectedness towards wild animals and, thus, elicit an empathetic reaction vis-à-vis their lifethreatening circumstances? The main objective of this study is to investigate whether virtual reality can be an affective machine aimed at conserving the biodiversity of Earth. Recent developments in technology have heightened the hope for teachers to be able to, for instance, give a biology class and transport all their students into the jungle without leaving the classroom. Teachers can employ these new digital techniques to immerse students in an interactive environment to enhance their curiosity about the world. Examples of applications of virtual reality in education are: (1) 'PanicLobster', which helps to simulate a speaker who is afraid to give a speech in public; (2) 'Verizon-STRIVR', which trains employees in dangerous situations; and (3) 'A Grand View of Learning', which allows making virtual global field trips with students all over the world without leaving the room. Most research on virtual reality innovations focuses on the effects of presence, telepresence, and immersion of the users. However, what is not yet clear is the impact of virtual reality innovations on cognitive and affective outcomes.

Previous research has established that virtual reality allows for an embodied experience, which refers to being able to experience sensing that one's virtual body actually is one's own biological body (Kilteni, Groten, \& Slater, 2012). Recent work has developed a virtual tree storytelling experiment with an embodied experience. This research allowed users to experience being another life-form, in this case, a tree (Liu, Qian, \& Evans, 2017). A simulation of being a tree was enhanced with external settings to increase realism with the experience (for instance, participants could experience a bird perching on its branches, or how one of its branches was cut by a lumberjack). However, no previous study has investigated the experience of virtual wild animals and the relationship with embodiment. The purpose of the current study will be to explore how the embodiment of a virtual wild animal can affect user's empathy, perceived pain, and immersion of the users. Empathy is defined as the ability to share the feelings and thoughts of other people; it facilitates the process of social interactions. Empathy can generate the ability to feel the pain of another person and it can also repress antisocial behaviors and aggression. In recent years, a new construct - dispositional empathy with nature (Tam, 2013) has been defined in terms of the dispositional tendency to understand and share the emotional experience of the natural world. Development of this tendency plays a crucial role in the context of environmentalism and for assessment of environmental educational programs. For example, when reading news about the effect of plastic waste on polluting the ocean, exemplified on the stomach contents of a deceased whale, reader can put themselves in the place of the affected animal.

To sum up, in our study we explore the efficacy of the game character appearance in virtual reality simulations to develop an empathetic and immersive experience where users can step into the 'shoes' of nature. Teachers and students can become a wild animal through virtual reality which makes it possible for them to move and see their body and arms as a virtual animal body. This way, virtual reality creates the appearance of a virtual body that allows for the exploration of a simulated natural environment, in line with the proposition of Sobel (1996): “...we must begin in empathy, by becoming the animals before we can save them" (Sobel, 1996). 


\section{Methods}

To investigate the influence of the design of the virtual character appearance, we set up an experiment with two experimental conditions and a control condition. The two experimental conditions of the game character appearance are: natural body (virtual beaver) and artificial body (virtual robotic beaver). The control condition contains a baseline amorphous body character.

We compare different experimental conditions, in order to identify design elements that are more affective (empathetic) and immersive for users. We used different types of animals (that have different body appearances) for comparing their effect on the users. During the experimental situations, psychological measures were collected using the dispositional empathy questionnaires such as the Interpersonal Reactivity Index (Davis, 1983) and some questions about conservation of the endangered species. After the virtual simulation experience, participants were asked to answer questions regarding their virtual experience, using the virtual reality sickness symptoms scales, an immersion questionnaire (Jennett, et al.), situational empathy questions, embodiment questionnaire, perceived-pain and perceived emotions questionnaire. Finally, we used a semi-structured interview to collect additional information about the user's experience and potential distress experienced during the task.

\subsection{Participants}

In total, we recruited 51 participants. The participants (between 18 to 35 years old, balanced for gender) came from the university student population in the Netherlands. The students received a course credit for their participation. The participants originated from Belarus (1), Brazil (1), Bulgaria (4), Chile (1), China (1), Colombia (2), Croatia (1), Curacao (1), Finland (1), France (1), Germany (1), Greece (1), Indonesia (1), Italy (2), Latvia (1), Lithuania (1), Moldova (1), Montenegro (1), Netherlands (21), Norway (1), Romania (2), Turkey (2), and United States (2). The research received ethical approval from the institutional Ethics Review Board of the Tilburg School of Humanities and Digital Sciences.

\subsection{Stimuli}

We designed an experimental game in virtual reality called 'Justin Beaver VR'. We used two different body designs for a humanoid virtual beaver (as the wild animal) with a natural and artificial appearance, and a control condition (amorphous figure). The three designs were: a natural body (Figure 1a), and an artificial body (Figure 1b) design, whereas the control condition uses an amorphous design (Figure 1c). 
a . Virtual beaver: Natural body

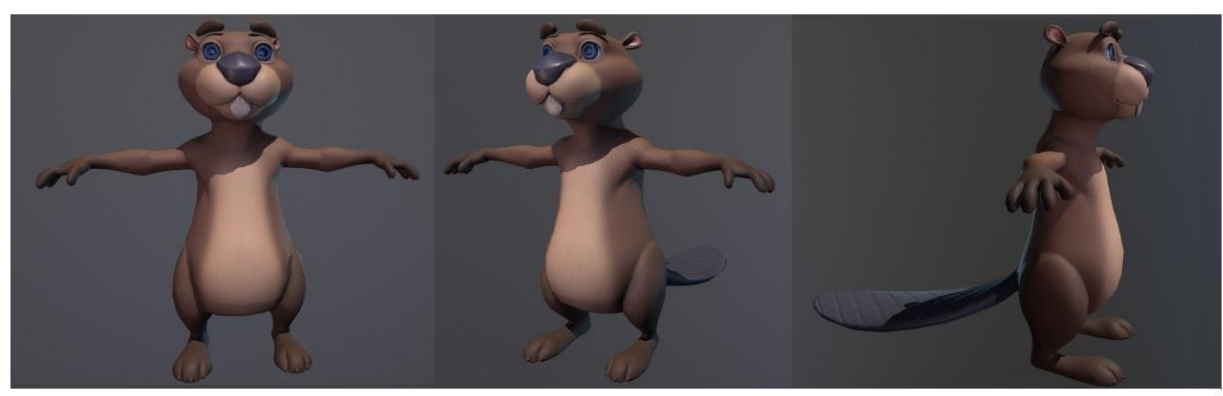

b. Virtual robot-beaver: Artificial body

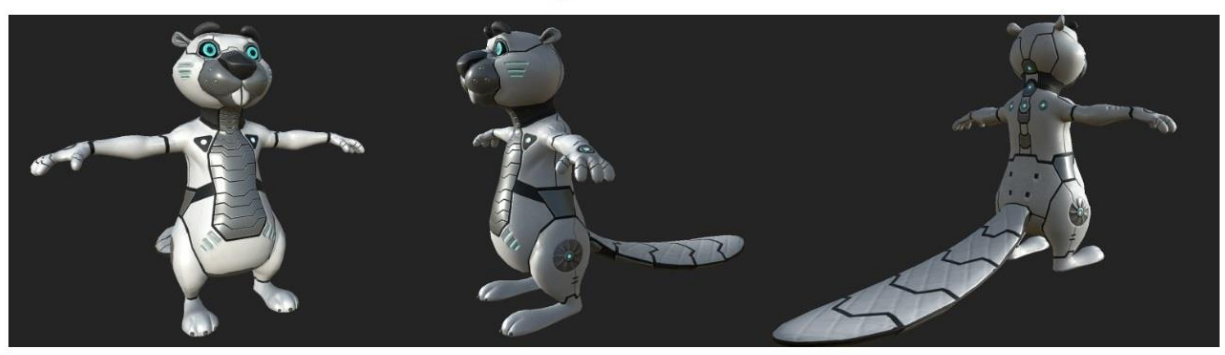

c. Figure: amorphous body

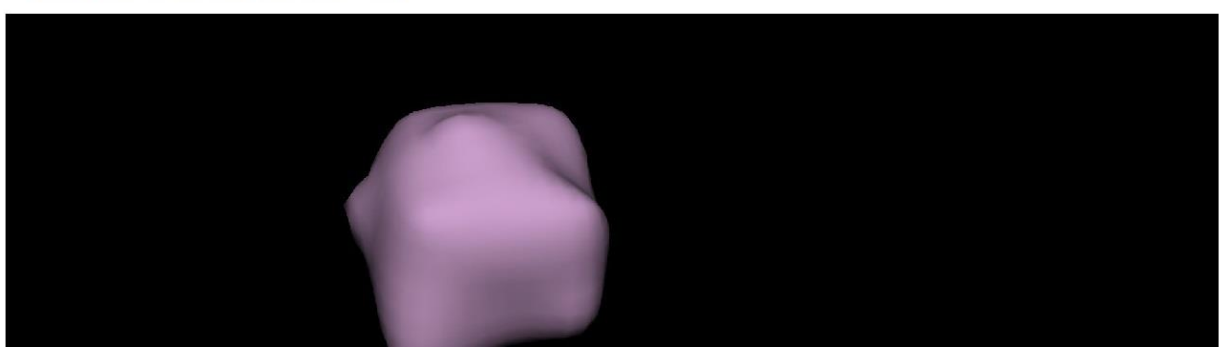

Figure 1 (a-c): Modeling in 3D design of the natural beaver (a), artificial beaver (b), and amorphous figure (c).

\subsection{Procedure}

The study uses a game in virtual reality, which is called "Justin Beaver VR". The beaver (i.e., the virtual body character) is the main character in the game. The game environment is presented to the participants in virtual reality using a head-mounted display (Oculus Rift). The game has three simulation scenes. In the first scene, the participants see the body of the virtual character (beaver, robot beaver or amorphous figure) as if they see their virtual body in front of the mirror for the duration of one minute (see Figure 2). The participants can examine their virtual appearance and appropriate the concept of their new body. The hands of the game character are connected to the Oculus Rift controllers, to simulate the movement of the limbs. The user can see a timer indicating the moment when the game starts. In the second scene, the experimental task consists of exploring the natural habitat of the animal by supplying the virtual character with energy (eating wood and fish from the ground), break time (sleeping in a house), and fun (playing with tree branches and swimming) in a virtual game lasting four minutes. Users receive visual and haptic feedback when their game character is swimming and playing with fish. In the last scene, situation-induced empathy is evoked by introducing a distress situation when 
another virtual character (a "hunter") shoots the virtual character. The users experience the shot as a mild simulated pain sensation from vibrational motors produced by a haptic virtual reality vest. We used the Tactot haptic virtual reality vest from Bhaptics. The distress situation has a duration of ca. 16 seconds. Following the distress situation, there is a delay of few seconds and then a screen appears with the message: "Game Over".
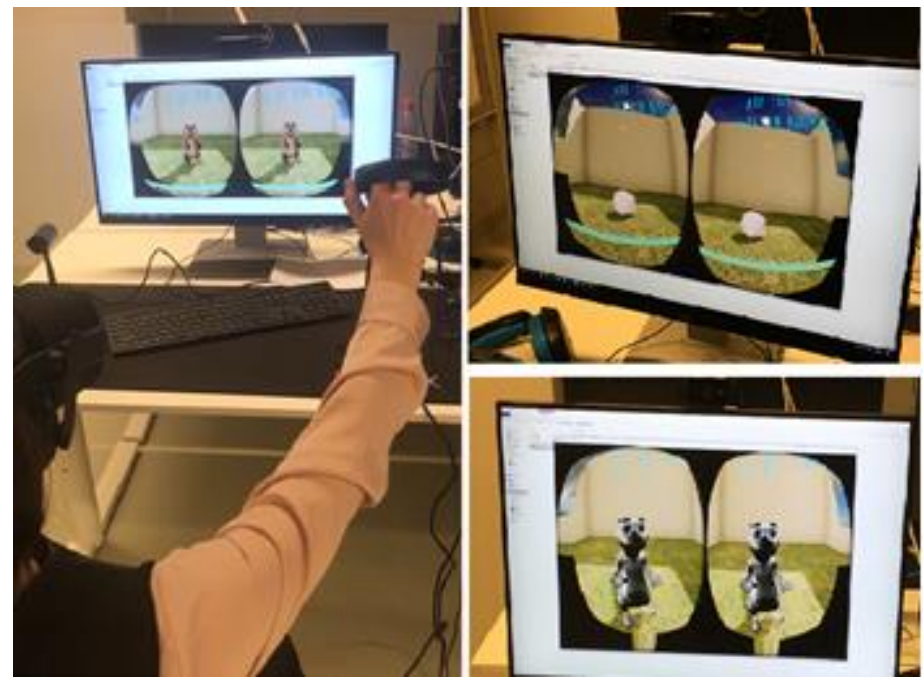

Figure 2: Virtual reality game experience.

At the beginning of each experiment, participants were provided with written and verbal instructions and information about the experiment. It was made clear to them that they could terminate the experimental session at any time without giving any reason. Testing started only once the participants provided their informed consent in writing. During each test session, the researcher monitored the participants continuously, taking special care with participants who have never experienced simulations in virtual reality. The researcher could terminate the experiment immediately in case any signs of potential distress were observed.

Before the game, the Interpersonal Reactivity Index (IRI) questionnaire and self-reported animal conservation tendency questionnaire, were administrated online. They took approximately 10 minutes to complete. After the gaming experience in virtual reality, the participants filled out an online immersion questionnaire, embodiment questionnaire, situational empathy questions, self-reported emotions, perceived-pain questionnaire, virtual reality sickness symptoms questionnaire [5], and selfreported animal conservation tendency questionnaire. This took approximately 20 minutes. Finally, the main experimenter (first author) conducted a semi-structured interview about emotional discomfort, taking about 5 minutes. In total, the experiment lasted approximately 40 minutes.

\subsection{Instrumentation}

2.4.1 Dispositional Empathy: To measure dispositional empathy, the participants filled out the "Interpersonal Reactivity Index (IRI)" questionnaire (Davis, 1983) which measures empathy on four sub-scales: perspective taking (the tendency to assume the psychological another opinion), fantasy (the tendency to connect to the feelings and actions of fictitious characters in movies, games and books), empathic concern (feelings of sympathy for the problems of other people), and personal distress ("self- 
oriented" feelings of personal concern and nervousness in tense interpersonal situations). The participants indicated their answers on a 5-point scale where the extremes were labeled $\mathrm{A}={ }^{\text {'Does not }}$ describe me well' and $\mathrm{E}=$ 'Describes me very well'. The test consisted of 28 items. The items (1-2, 46, 8-11, 16-17, and 20-28) were scored: $A=0, B=1, C=2, D=3, E=4$. The reversed-scored items of this test were: $3,7,12,13,14,15$, and 19. The Cronbach's alpha for the IRI questionnaire was reliable with $\alpha=0.862$.

2.4.2 Self-reported situational empathy: We measured self-reported situational empathy with a single item: 'To what extent did you empathize with the character (animal/robot-animal/amorphous figure)?' on a 5-point scale where the extremes were labeled ( $1=$ 'Not at all' and $5=$ 'Very much').

2.4.3 Immersion: We measured immersion using a questionnaire that was originally developed by Jennett, et al. (2008). The participants indicated their answers on a 5-point scale where the extremes were labeled $1=$ 'Not at all' and $5=$ 'Very much'. The test consisted of 31 items. The items 1-5, 7, 1117, 19, and 21-31 were scored: 1 to 5. The reversed-scored items of this test were: $6,8,9,10,18$, and 20. The Cronbach's alpha for the Immersion questionnaire was reliable with $\alpha=0.805$.

2.4.4 Virtual Reality Sickness Symptoms: We measured the virtual reality sickness symptoms using a 9-item questionnaire originally developed by Lu (2016). The participants indicated their answers on a 5-point scale where the extremes were labeled $1=$ 'None' and $5=$ 'Severe'. This questionnaire was applied after the game experience. The Cronbach's alpha for the Virtual Reality Sickness Symptoms was reliable with $\alpha=0.801$.

2.4.5 Self-reported emotions: We measured self-report emotions with a single item: 'What did you feel when the game character died?'. The participants were instructed to choose more than one emotion, if applicable. The list of emotions included the following options: joy, happiness, surprise, anger, sadness, fear, disgust, nothing, and other.

2.4.6 Self-reported animal conservation tendency questionnaire: We measured animal conservation tendency with four items: (1) 'I am really fond (like) of animals', (2) 'I am interested in protecting endangered species', (3) 'I like the animal on the picture', and (4) 'I am interested in protecting the animal on the picture'. The participants indicated their answers on a 7-point scale where the extremes were labeled $1=$ 'Strongly disagree' and $7=$ 'Strongly agree'. This questionnaire was applied before and after the game experience. The Cronbach's alpha for the self-reported animal conservation tendency was reliable with $\alpha=0.867$.

2.4.7 Perceived-pain questionnaire: We measured perceived pain based on Das, Grimmer, Sparnon, McRae, and Thomas (2005), with a single item: 'Which picture best depicts how the virtual shot made you feel?'. The participants indicated their answers on a 5-point scale (see Figure 3).

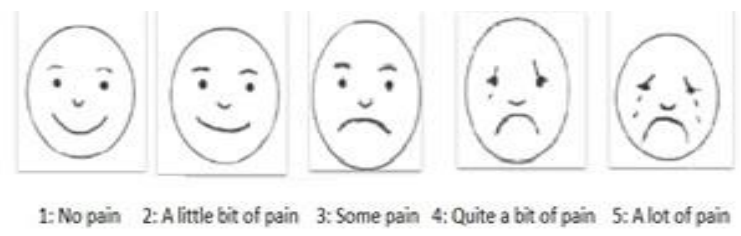

Figure 3: Images of the perceived-pain questionnaire. 
2.4.8 Embodiment questionnaire: We measured immersion using a modified version of the questionnaire that was originally developed by Wilson (2008). Participants indicated their answers on a 7 -point scale where the extremes were labeled $1=$ 'Strongly disagree' and $7=$ 'Strongly agree'. The test consisted of 25 items. The reversed-scored items were: 2, 4, 9, and 15. The Cronbach's alpha for the Immersion questionnaire was reliable with $\alpha=0.904$.

\subsection{Potential ethical threats during data collection}

The users experienced a simulated pain sensation from the vibrational motors produced by the haptic virtual reality vest. The simulated pain sensation never exceeded a participant's tolerance limit and participants were able to escape or terminate the painful stimulus at any point during the experiment. There were no safety hazards related to the use of the virtual reality vest. Non-intrusive sensing technologies collecting physiological and behavioral data were used. These techniques have also been used in abundance by other research projects. To ensure participants' comfort and safety we excluded participants with a history of migraine, epilepsy, and currently pregnant females. Also, participants were able to withdraw from the study at any time without giving a reason. Participants were informed about the physiological measurements collected during the experiment and could indicate for which purpose they approved these measurements to be used. The participants signed the corresponding consent form prior to the beginning of the experiment. The participants received a phone number and email of the university psychologist to contact in case they experienced any discomfort following the experiment. The approval of the ethics committee for this experiment has the reference REDC\#2017/01, with the titled "Effect of Game Character on empathy and immersion".

\section{Results}

Prior to the testing of experimental hypotheses, we ran a series of ANOVAs to check if the participants in the three different experimental conditions differed with respect to their dispositional empathic tendencies. There was no significant difference with respect to IRI and its sub-scales in the three conditions (see Table 1), with the exception of Fantasy (IRI_FS). Since Fantasy is the least relevant sub-scale of IRI for the purposes of this research that focuses on the link between empathy and character appearance, we disregarded it in the subsequent analyses.

\begin{tabular}{cccccc}
\hline Source & $\begin{array}{c}\text { Sum } \\
\text { of Squares }\end{array}$ & $\boldsymbol{D} \boldsymbol{f}$ & $\boldsymbol{\eta}$ & $\boldsymbol{p}$ \\
\hline Dispositional Empathy & 0.404 & 2 & 0.934 & 0.037 & 0.400 \\
\hline IRI_PT & 0.438 & 2 & 0.615 & 0.025 & 0.545 \\
\hline IRI_FS & 3.957 & 2 & 4.267 & 0.151 & 0.020 \\
\hline IRI_EC & 0.745 & 2 & 0.890 & 0.036 & 0.417 \\
\hline IRI_PD & 0.529 & 2 & 0.605 & 0.025 & 0.550 \\
\hline
\end{tabular}

Table 1: One-way ANOVA on the effects of character appearance on empathy

Subsequently, we tested our expectations regarding the effect of experimental conditions on the situational variables. The bar chart in Figure 4 represents the proportion of each emotion reported by participants when the game character died, split into conditions by the appearance of the game character (virtual beaver, virtual robot-beaver, or amorphous figure). It can be seen that the emotions more frequently reported during distress event were surprise and sadness. Interestingly, anger and disgust were reported only in the conditions with the virtual robot animal and the amorphous figure. 


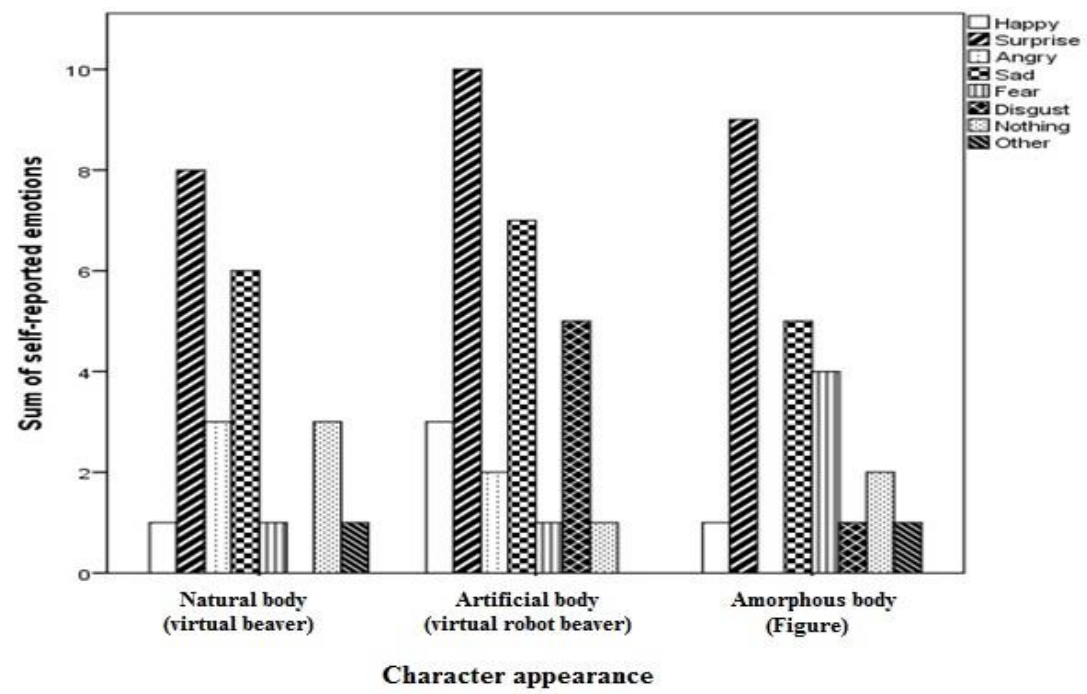

Figure 4: Bar chart of the number of each emotion reported by participants during distress situation.

Results of the Pearson correlation (see Table 2) indicated that there was a significant positive association between perceived pain when the game character was shot with dispositional empathy $(r \quad 51)=0.428, p=0.002)$, empathic concern $(r \quad(51)=0.456, p=0.001)$, immersion $(r(51)=0.419, p=0.002)$, and embodiment $(r(51)=0.396, p=0.004)$.

Situational empathy toward game character had a significant positive correlation with immersion $(r(51)=0.559, p<0.001)$, and embodiment $(r(51)=0.396, p=0.004)$; immersion and embodiment were also positively correlated with each other, $(r(51)=0.601, p<0.001)$.

\begin{tabular}{|c|c|c|c|c|c|c|c|c|c|c|c|}
\hline Variables & $\mathbf{M}$ & SD & 1 & 2 & 3 & 4 & 5 & 6 & 7 & 8 & 9 \\
\hline $\begin{array}{l}1 \text { Dispositional } \\
\text { Empathy }\end{array}$ & 2.39 & 0.46 & - & & & & & & & & \\
\hline $\begin{array}{l}2 \text { IRI_Perspective } \\
\text { Talking }\end{array}$ & 2.62 & 0.59 & $0.595^{* *}$ & - & & & & & & & \\
\hline 3 IRI_Fantasy & 2.49 & 0.72 & $0.761^{* *}$ & 0.200 & - & & & & & & \\
\hline $\begin{array}{l}4 \text { IRI_Empathic } \\
\text { Concern }\end{array}$ & 2.71 & 0.65 & $0.877^{* *}$ & $0.582^{* *}$ & $0.567^{* *}$ & - & & & & & \\
\hline $\begin{array}{l}5 \text { IRI_Personal } \\
\text { Distress }\end{array}$ & 1.75 & 0.66 & $0.591^{* *}$ & -0.010 & $0.312^{*}$ & $0.346^{*}$ & - & & & & \\
\hline $\begin{array}{l}6 \text { Situational } \\
\text { Empathy }\end{array}$ & 3.14 & 1.22 & 0.090 & 0.040 & -0.100 & 0.150 & 0.180 & - & & & \\
\hline 7 Perceived Pain & 1.94 & 1.01 & $0.428^{* *}$ & $0.344^{*}$ & 0.210 & $0.456^{* *}$ & 0.220 & 0.120 & - & & \\
\hline 8 Immersion & 3.15 & 0.42 & 0.130 & 0.110 & -0.050 & 0.250 & 0.080 & $0.559^{* *}$ & $0.419^{* *}$ & - & \\
\hline 9 Embodiment & 3.64 & 0.88 & 0.270 & 0.200 & 0.120 & $0.305^{*}$ & 0.150 & $0.396^{* *}$ & $0.374^{* *}$ & $0.601^{* *}$ & - \\
\hline
\end{tabular}

Table 2: Bivariate correlations between the measured variables. 
To measure the potential effect of character appearance on animal conservation tendency, we calculated a new variable as the difference between the post and pre-test of the conservation animals. A multivariate analysis of variance (MANOVA) was conducted to assess the influence of the game character appearance on animal conservation tendency situational empathy, perceived pain, immersion, and avatar embodiment: $\mathrm{F}(5,45)=2.387, p=0.053, \eta_{p}{ }^{2}=0.210$.

We found a statistically significant effect of appearance on perceived pain, $\mathrm{F}(2,48)=4.405, p=0.018, \eta_{p}{ }^{2}=0.155$. Follow-up comparisons indicated a significant difference between the condition with a natural body (virtual beaver) $(M=2.35, S D=0.27)$ and the condition with the amorphous figure (control condition) $(M=1.41, S D=0.7), p=0.015$, but not a significant difference between the natural body (virtual beaver) and the artificial body (virtual robot beaver) $(M=2.06, S D=0.74), p=0.639$ (see Figure 5).

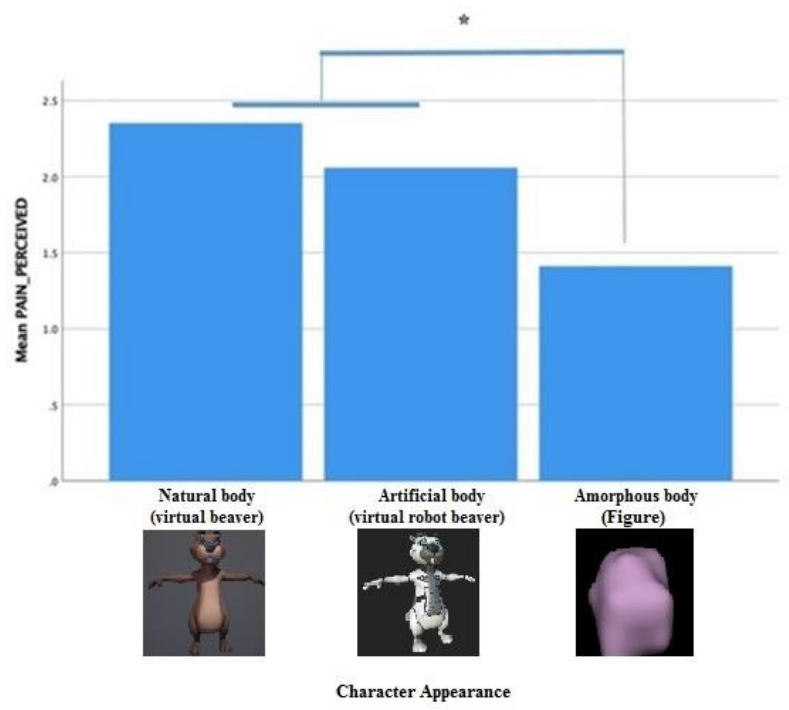

Figure 5: A bar chart of the mean perceived pain in relation to the game character appearance.

Next to that, we found a statistically significant effect of appearance on immersion, $\mathrm{F}(2,48)=4.275, p=0.020, \eta_{p}{ }^{2}=0.151$. Follow-up comparisons indicated a significant difference between the condition with the natural body (virtual beaver) $(M=3.37, S D=0.40)$ and artificial body (virtual robot beaver) $(M=3.04, S D=0.42), p=0.044$ and the amorphous figure (control condition) $(M=3.02, S D=0.35), p=0.034$. Finally, there was no significant effect of the game character appearance on situational empathy $\mathrm{F}(2,48)=1.007, p=0.373, \eta_{p}{ }^{2}=0.040$, and embodiment $\mathrm{F}(2,48)=0.631, p=0.536, \eta_{p}^{2}=0.026$.

\section{Conclusions}

In our research, we explored the effects of designing a game character resembling a virtual animal with natural body or a virtual robotic animal with artificial body on a number of constructs related to players' emotional reactions towards the character. We found that a game character that resembled a virtual animal with natural body elicited the highest degree of immersion in a first-person virtual reality game. Using a virtual animal with natural body or a virtual robotic animal with artificial body also increased the perceived pain reported by participants after they experienced a distressing physical event 
concerning the character. There were no effects of game character appearance on situational empathy and embodiment. We also did not observe any effect on self-reported animal conservation tendency. In general, however, participants reported experiencing surprise and sadness when the game character was shot, independently of the experimental condition. Finally, we observed a significant positive association between dispositional empathy, measured with the standard IRI questionnaire, and perceived pain related to the distress event in the game. In a follow-up study, we intend to compare the effect of a first-person experience of the 3D virtual environment to a third-person experience.

Acknowledgements: We thank to Esteban Plazas, Julian Andres Ramirez, and Johnny Alejandro Sierra for their help in the design and development of this study.

\section{References}

Carlisle, D. (2010, April). graphicx: Enhanced support for graphics. Retrieved from http://www.ctan.org/tex-archive/ help/Catalogue/entries/graphicx.html

Das, D., Grimmer, K., Sparnon, A., McRae, S., \& Thomas, B. (2005). The efficacy of playing a virtual reality game in modulating pain for children with acute burn injuries: a randomized controlled trial [ISRCTN87413556]. BMC pediatrics, 1. Retrieved from https://bmcpediatr.biomedcentral.com/articles/10.1186/1471-2431-5-1

Davis, M. (1983). Measuring individual differences in empathy: Evidence for a multidimensional approach. Journal of personality and social psychology, 44(1), 113-126. doi:http://dx.doi.org/10.1037/0022-3514.44.1.113

Earthday.org. (2018). Fact Sheet: Global Species Decline. Retrieved from https://www.earthday.org/2018/05/18/fact-sheet-global-species-decline/

Jennett, C., Cox, A. L., Cairns, P., Dhoparee, S., Epps, A., Tijs, T., \& Walton, A. (n.d.). Measuring and defining the experience of immersion in games. International journal of human-computer studies, 66(9), 641-661. doi:https://doi.org/10.1016/j.jijcs.2008.04.004

Kilteni, K., Groten, R., \& Slater, M. (2012). The sense of embodiment in virtual reality. Presence: $\begin{array}{llll}\text { Teleoperators and } & \text { Birtual }\end{array}$ doi:https://doi.org/10.1162/PRES_a_00124

Liu, X., Qian, Y., \& Evans, M. (2017). If you were a tree. Virtual reality opens up a novel perspective in storytelling and engagement. It's what seeded our VR film projects TreeSense and Tree. Retrieved from https://www.media.mit.edu/posts/tree-treesense/

Lu, D. (2016). Virtual Reality sickness during immersion: An investigation ofpotential obstacles towards general accessibility of VR technology. Uppsala University, Disciplinary Domain of Humanities and Social Sciences, Faculty of Social Sciences, Department of Informatics and Media. Retrieved from http://www.divaportal.org/smash/record.jsf?pid=diva2\%3A1129675\&dswid=4473

Miller, J. (2005). Biodiversity conservation and the extinction of experience. Trends in ecology \& evolution, 20(8), 430-434. doi:https://doi.org/10.1016/j.tree.2005.05.013

Sobel, D. (1996). Beyond ecophobia. (O. Society, Ed.) Great Barrington, MA. Retrieved from http://www.simplicityparenting.com/Beyond\%20Ecophobia.pdf

Tam, K. (2013). Dispositional empathy with nature., 35, 92104. Journal of Environmental Psychology, 35, 92-104. doi:https://doi.org/10.1016/j.jenvp.2013.05.004 